\title{
Groups for Undergraduates
}


This page is intentionally left blank 


\section{GROUPS FOR} UNDERGRADUATES

\section{John Atwell Moody}

Warwick University

United Kingdom 
Published by

World Scientific Publishing Co. Pte. Ltd.

P O Box 128, Farrer Road, Singapore 9128

USA office: Suite 1B, 1060 Main Street, River Edge, NJ 07661

UK office: P O Box 973, London N12 7JS

\section{GROUPS FOR UNDERGRADUATES}

Copyright () 1994 by World Scientific Publishing Co. Pte. Ltd.

All rights reserved. This book, or parts thereof, may not be reproduced in any form or by any means, electronic or mechanical, including photocopying, recording or any information storage and retrieval system now known or to be invented, without written permission from the Publisher.

For photocopying of material in this volume, please pay a copying fee through the Copyright Clearance Center, Inc., 27 Congress Street, Salem, MA 01970, USA.

ISBN 981-02-1557-6

ISBN 981-02-2105-3 (pbk)

Printed in Singapore by Uto-Print 


\section{Contents}

Preface

Author's message to the student ix

Chapter 0. Introduction 1

Representations (basic definitions) - Classification of permutation representations

Chapter 1. Number-Theoretic Preliminaries

Binomial theorem-The Chinese Remainder TheoremSylow theorems-The structure of $\mathbb{Z} / n \mathbb{Z}^{\times}$-Finite FieldsThe conjugacy classes in $G l_{m}(\mathbb{Z} / p \mathbb{Z})$-Polynomial factorization and finite fields

\section{Chapter 2. Semidirect Products}

Chapter 3. Presentations

Presentations of semidirect products-Example: groups of order $75-$ The examples of $S_{n}, U_{n}$, groups of order $p^{3}$-Principle relating homomorphisms and presentationsTietze moves

Chapter 4. Calculation of a List of the Groups of Order 1-31 
Chapter 5. Rewriting and Transfer

Rewriting-Example: $A_{n}$-Action on relation subgroup-

Transfer-Burnside's $p$-complement theorem-Calculation of a list of groups of order 33-41 using transfer

Chapter 6. Classification of Certain Groups

Chapter 7. Cohomology

Chapter 8. More Cohomology

The coprime-order theorem-Application: the groups of order 42

Chapter 9. Linear Representations

The proof that they are unitary-Mashke's theorem-

Schur's lemma-Unique decomposition into irreduciblesInduced representations

Chapter 10. Construction of the Linear Representations 147

Tensor products-Further cohomology-Classification theorem

Chapter 11. Further Cohomology 158

Vanishing lemma-Higher transfer

Chapter 12. Calculation of a List of the Representations of the Groups of Order 1-12 161 


\section{Preface}

This text contains everything which you'll need to discover and construct for yourself all the $n$-element matrix groups or permutation groups, for any reasonably small value of $n$, and for many large values too. In order to begin reading, you should already know about the following things: subgroups, homomorphisms, normal subgroups, cosets, Lagrange's theorem, conjugate subgroups, the isomorphism theorem, and the structure of finite abelian groups. Otherwise the text is selfcontained.

Some conventional tools, such as characters, aren't useful for us, as they are too blunt to determine the actual matrices which make up the elements of our groups. Other tools such as transfer, cohomology, and induction, often used to prove existence (or otherwise) of certain groups or representations, must here play a role in their actual construction.

In the text I'll go through the construction of the abstract groups of order 1-31 and 33-42 - in case you get stuck at an embarrassingly early stage - and towards the end I'll go through the classification of the matrix representations of the ones of order less than 13. There was no particular reason for stopping at these numbers, and, in particular, the tables of matrix representations can be quite easily extended to cover the groups of order 1-31, and many more.

Throughout, we'll build our groups and their representations inductively, starting with cyclic groups. This works because the only groups we'll encounter which can't be factor- 
ize (ie. with no proper nontrivial normal subgroups) will be cyclic of prime order. There do exist other so-called simple groups, the smallest being the 60 -element group $A_{5}$; and, to progress further one has to classify the abstract simple groups and their representations. The first of these tasks has been completed, and the second is in progress.

The exercises in the text serve a slightly different purpose than you may be used to. They mainly ask you to fill-in the occasional gap in a proof, or to consider an issue from another point of view than the one I've chosen. In addition, you should imagine you are assigned the following infinite sequence of unsolved exercises:

Exercise n.m. Construct all the groups consisting of $n$ distinct $m$-by- $m$ matrices, up to change of basis. In addition, construct all groups consisting of $n$ distinct permutations of $\{1,2, \ldots, m\}$, up to renumbering. 


\section{Author's message to the student}

If you are a beginning Mathematics student, or a student whose major interest lies outside Mathematics the experience you'll have reading this book may be firstly one of pleasure. As interesting structures appear to unfold from just a few axioms, you may initially hope, as past students have, to completely understand the finite matrix groups, for instance. In time, though, when no final answer emerges, you may begin to wonder: Weren't the beautifully simple theorems of this subject enough? If not, why have I been asked to read this book?

Although I can't answer these questions for you, I would like to tell you why I wrote such a book.

It is possible for an axiom to be true, and its consequences meaningfully exploited, precisely because the brain is a biological organ. A child knows how to deduce for himself the consequences of an axiom told to him by his father or his teachers. As a child, living in a Maine village, I remember my father telling me, "You can eat anything which comes from the sea."

Yet, the underlying reason why it is possible for a few rules to imply many meaningful things is because the mind only needs hints or clues to recognize which way to live in one or another niche in the natural world. Once the appropriate clues are present, one innately behaves in the appropriate way. Once a child is told, "You may eat apples," he will also, without being told, discard the cores, planting new apple trees in the process.

I think, when mathematicians first began travelling and 
meeting, there was a feeling that groups could be well understood, and that understanding them would lead to a good understanding of equations, and many other things. The fact that groups can't be understood, and the obvious fact that their understanding cannot carry any meaningful information, are both important to confront.

Such facts are important to confront because there still exist people who act as though science can carry meaningful information divorced from nature. For instance, you know that there must be an intricate but unknown correspondence between tastes of foods and their constituent nutrients, by which we choose just the balance of nutrients we need. Yet scientists are busy obtaining the tastes we like, without regard to this crucial correspondence, just by genetically altering the tastes. There are many more serious examples too.

In conclusion, I hope that, upon learning - possibly through this text - that even the most elementary mathematical problems typically end up being insoluble, you will think about why this is so. I hope that, aware of the limitations of abstract science, you may attempt to shun its powerful but necessarily short-sighted applications in industry. 